\title{
Identification of HRAS mutations and absence of GNAQ or GNA11 mutations in deep penetrating nevi
}

\author{
Ryan P Bender ${ }^{1}$, Matthew J McGinniss ${ }^{2}$, Paula Esmay ${ }^{1}$, Elsa F Velazquez ${ }^{3,4}$ and \\ Julie DR Reimann ${ }^{3,4}$ \\ ${ }^{1}$ Caris Life Sciences, Phoenix, AZ, USA; ${ }^{2}$ Genoptix Medical Laboratory, Carlsbad, CA, USA; \\ ${ }^{3}$ Dermatopathology Division, Miraca Life Sciences Research Institute, Newton, MA, USA and \\ ${ }^{4}$ Department of Dermatology, Tufts Medical Center, Boston, MA, USA
}

\begin{abstract}
HRAS is mutated in $\sim 15 \%$ of Spitz nevi, and GNAQ or GNA11 is mutated in blue nevi $(46-83 \%$ and $\sim 7 \%$ respectively). Epithelioid blue nevi and deep penetrating nevi show features of both blue nevi (intradermal location, pigmentation) and Spitz nevi (epithelioid morphology). Epithelioid blue nevi and deep penetrating nevi can also show overlapping features with melanoma, posing a diagnostic challenge. Although epithelioid blue nevi are considered blue nevic variants, no GNAQ or GNA11 mutations have been reported. Classification of deep penetrating nevi as blue nevic variants has also been proposed, however, no GNAQ or GNA11 mutations have been reported and none have been tested for HRAS mutations. To better characterize these tumors, we performed mutational analysis for GNAQ, GNA11, and HRAS, with blue nevi and Spitz nevi as controls. Within deep penetrating nevi, none demonstrated GNAQ or GNA11 mutations (0/38). However, $6 \%$ revealed HRAS mutation (2/32). Twenty percent of epithelioid blue nevi contained a GNAQ mutation (2/10), while none displayed GNA11 or HRAS mutation. Eighty-seven percent of blue nevi contained a GNAQ mutation (26/30), 4\% a GNA11 mutation (1/28), and none an HRAS mutation. Within Spitz nevi, none demonstrated GNAQ or GNA11 mutations (0/30). Seventeen percent contained an HRAS mutation (5/30). All GNAQ and GNA11 mutations were p.Q209L (c.626A $>$ T) point mutations, except 2 GNAQ mutations, which contained novel c.625_626CA $>$ TT double mutations. Four HRAS mutations were in exon 2, and three in exon 3 . This is the first study to identify HRAS mutations in deep penetrating nevi. The presence of HRAS mutations and absence of GNAQ or GNA11 mutations in deep penetrating nevi suggests classification of these unusual nevi within the Spitz nevus category of melanocytic tumors, rather than the blue nevus category.

Modern Pathology (2013) 26, 1320-1328; doi:10.1038/modpathol.2013.77; published online 19 April 2013
\end{abstract}

Keywords: blue nevus; deep penetrating nevus; epithelioid blue nevus; GNA11; GNAQ; HRAS; spitz nevus

Melanocytic nevi with epithelioid cytomorphology include epithelioid and spindle cell (Spitz) nevi, epithelioid blue nevi, and deep penetrating nevi. Deep penetrating nevus, a term first coined by Seab et $a l_{1}{ }^{1}$ are unusual melanocytic neoplasms difficult to classify. Deep penetrating nevi are uncommon but are of importance due to their histological and clinical overlap with melanoma. They most commonly present on the extremities, followed by the head and neck and upper trunk. ${ }^{2}$ The age range at presentation is wide; however, as with Spitz nevi,

Correspondence: Dr JDR Reimann, MD, PhD, Dermatopathology Division, Miraca Life Sciences Research Institute, 320 Needham Street, Suite 200, Newton, MA 02464, USA.

E-mail: jreimann@MiracaLS.com

Received 30 January 2013; revised 6 March 2013;

accepted 7 March 2013; published online 19 April 2013 deep penetrating nevi most commonly present in younger patients (adolescence and young adults). They typically present as predominantly dermal to focally compound melanocytic proliferations with extension into the reticular dermis particularly along adnexal structures and neurovascular bundles, often extending into the subcutis. They tend to have a relatively symmetric wedge-shaped nodular growth pattern, although superficial variants can occur. ${ }^{2}$ A plexiform disposition is also often present. Deep penetrating nevi are composed of epithelioid melanocytes with abundant finely pigmented cytoplasm with associated pigment-laden macrophages. There is often a minor component of banal nevocellular nevus admixed with the deep penetrating nevus component (so-called 'combined nevus'). Random cytological atypia can be present in deep penetrating nevi, 
as can occasional dermal mitoses (typically not $>2$ mitoses per $\mathrm{mm}^{2}$ ). Despite their sometimes histologically alarming features, recurrence is uncommon and malignant transformation is exceptional in conventional deep penetrating nevi. ${ }^{2}$ Deep penetrating nevi with atypical features (asymmetry, pleomorphism, $>2$ mitoses per $\mathrm{mm}^{2}$, prominent nucleoli, expansile growth, and/or an inflammatory host response) can be difficult to distinguish from melanoma on histological grounds. Moreover, there have been reports of atypical deep penetrating nevi with locoregional spread to lymph nodes. ${ }^{3-7}$ Given the latter data and ambiguous histology, atypical deep penetrating nevi are often regarded as having uncertain malignant potential. ${ }^{2}$ Deep penetrating nevi are considered by some authors to fall within the category of blue nevus, ${ }^{8}$ due to some overlapping features, including heavy pigmentation, predominantly dermal growth pattern, and frequent HMB45 positivity. The possibility of subclassification into the Spitz nevus category has also been raised, due primarily to the presence of epithelioid cytomorphology in deep penetrating nevi. However, little research has been done at the molecular level to further characterize and subclassify these lesions. ${ }^{9-11}$

Epithelioid blue nevi were initially described most commonly in association with Carney Complex. ${ }^{12}$ Subsequently, similar lesions have been reported in patients without Carney Complex under various different names, including epithelioid blue nevi, 'animal-type melanoma', 'pigment synthesizing melanoma', and more recently 'pigmented epithelioid melanocytoma' (the latter a term proposed to encompass all three previously mentioned categories due to the significantly overlapping histopathological features and clinical behavior of the three entities ${ }^{13}$ ). They most commonly present on the extremities and trunk but can occur at any site. The age range at presentation is wide, although presentation in young adulthood is most common. ${ }^{13}$ Microscopically, epithelioid blue nevi are heavily pigmented dermal melanocytic proliferations usually with infiltration of dermal collagen at the periphery and occasional extension into the subcutis. They are composed of three cell types; pigmented round epithelioid melanocytes with prominent nucleoli, pigmented dendritic melanocytes, and scattered larger epithelioid cells with abundant less pigmented cytoplasm. Melanophages are also present. Occasional mitoses (typically $<3$ per $\mathrm{mm}^{2}$ ) can be seen. The concept of epithelioid blue nevus/pigmented epithelioid melanocytoma as a low-grade melanoma/borderline tumor of unpredictable malignant potential is based on several case series demonstrating that while a subset of these lesions have metastasized (predominantly locoregionally), none has resulted in death, with a median clinical follow-up period of 5 years. ${ }^{14}$ No histological features distinguish non-metastasizing lesions from those with metastatic potential.
Cutaneous melanocytic lesions, both benign and malignant, have been shown to harbor frequent activating mutations in the MAP kinase signaling pathway. For example, genetic analysis of Spitzoid melanocytic proliferations has revealed that approximately $15 \%$ of Spitz nevi harbor mutations in HRAS, typically in either exon 2 (codon 12 or 13) or exon 3 (codon 61 ). ${ }^{15-19}$ This is in contrast to all spitzoid melanomas and conventional melanomas that have been analyzed to date, in which HRAS mutations are rare. ${ }^{17,19,20}$ In addition, $B R A F$ and NRAS mutations are uncommon in Spitz nevi, whereas they are common in melanomas. ${ }^{21}$ Thus the presence of an HRAS mutation in a melanocytic neoplasm with Spitzoid morphology appears to favor the diagnosis of Spitz nevus, rather than a Spitzoid melanoma. To date, neither epithelioid blue nevi nor deep penetrating nevi have been analyzed for HRAS mutations in the literature.

Mutational analysis by sequencing has revealed that either GNAQ or GNA11 is frequently mutated in uveal melanomas and in blue nevi. ${ }^{10,11}$ GNAQ and GNA11 are GTP-binding proteins with a role in MAP kinase pathway activation. GNAQ and GNA11 have a role in dark skin pigmentation as evidenced by an increase in dermal melanocytes in the setting of GNAQ- or GNA11-activating mutations, ${ }^{22}$ and thus GNAQ and GNA11 mutations in darkly pigmented dermal melanocytic tumors (ie, blue nevi, uveal melanoma, malignant blue nevi) are not surprising. To date, analysis of GNAQ, GNA11 or HRAS mutational status in epithelioid blue nevi has not been reported. Although GNAQ and GNA11 mutational status of a small sample set of Spitz nevi and deep penetrating nevi (19 Spitz nevi and 27 deep penetrating nevi) ${ }^{10,11}$ has been assessed in the literature, none demonstrated GNAQ or GNA11 mutation. (Table 1).

To better characterize these uncommon and sometimes diagnostically challenging melanocytic tumors, we performed mutational analysis for HRAS, GNAQ, and GNA11 on deep penetrating nevi and epithelioid blue nevi.

\section{Materials and methods}

Cases diagnosed as deep penetrating nevi and epithelioid blue nevi between 2005 and 2011 were retrieved from our case files at Miraca Life Sciences, Newton, MA, USA. Cases diagnosed as Spitz nevi and blue nevi between 2009 and 2010 were also retrieved as controls. All the diagnoses were independently confirmed by two dermatopathologists (JR and EV) as meeting definitive criteria for each of the diagnostic categories (Spitz nevus, epithelioid blue nevus, deep penetrating nevus, blue nevus). Those that did not meet stringent criteria, or where there was disagreement as to the diagnosis, were excluded. Criteria for the diagnosis of deep penetrating nevus were: primarily intradermal location 
Table 1 GNAQ and GNA11 mutation analysis of nevi: list of references

\begin{tabular}{|c|c|c|c|c|c|}
\hline Reference & Lesion type & $\begin{array}{l}\text { Number of GNAQ } \\
\text { Q209-mutated } \\
\text { samples }\end{array}$ & $\begin{array}{c}\text { Number of GNA11 } \\
\text { Q209-mutated } \\
\text { samples }\end{array}$ & $\begin{array}{l}\text { Number of GNAQ } \\
\text { R183-mutated } \\
\text { samples }\end{array}$ & $\begin{array}{c}\text { Number of GNA11 } \\
\text { R183-mutated } \\
\text { samples }\end{array}$ \\
\hline Lamba et al (2009) ${ }^{31}$ & Blue nevus & $6(n=13)$ & - & - & - \\
\hline \multirow[t]{6}{*}{$\begin{array}{l}\text { Van Raamsdonk et al } \\
(2009)^{10}\end{array}$} & $\begin{array}{l}\text { Malignant blue } \\
\text { nevus }\end{array}$ & $1(n=2)$ & $0(n=2)$ & - & - \\
\hline & Spitzoid melanoma & $0(n=2)$ & $0(n=2)$ & - & - \\
\hline & Blue nevus & $24(n=29)$ & $0(n=29)$ & - & - \\
\hline & Nevus of Ota & $1(n=17)$ & $0(n=17)$ & - & - \\
\hline & $\begin{array}{l}\text { Deep penetrating } \\
\text { nevus }\end{array}$ & $0(n=16)$ & $0(n=16)$ & - & - \\
\hline & Spitz nevus & $0(n=8)$ & $0(n=8)$ & - & - \\
\hline \multirow{5}{*}{$\begin{array}{l}\text { Van Raamsdonk et al } \\
(2010)^{11}\end{array}$} & Blue nevi & $76(n=133)$ & $9(n=133)$ & $1(n=93)$ & $1(n=93)$ \\
\hline & $\begin{array}{l}\text { Malignant blue } \\
\text { nevus }\end{array}$ & $2(n=6)$ & $1(n=6)$ & $0(n=3)$ & $0(n=3)$ \\
\hline & $\begin{array}{l}\text { Deep penetrating } \\
\text { nevus }\end{array}$ & $0(n=27)$ & $0(n=27)$ & $0(n=14)$ & $0(n=14)$ \\
\hline & Spitz nevus & $0(n=10)$ & $0(n=10)$ & $0(n=8)$ & $0(n=8)$ \\
\hline & Atypical Spitz tumor & $0(n=20)$ & $0(n=20)$ & $0(n=8)$ & $0(n=8)$ \\
\hline Emley et al (2011) ${ }^{32}$ & Blue nevus & $9(n=35)$ & - & - & - \\
\hline
\end{tabular}

within the reticular dermis; plexiform growth pattern, and a wedge-shaped silhouette; epithelioid melanocytes with vesicular chromatin and clear or finely pigmented cytoplasm; extension along neurovascular and/or adnexal structures; no or occasional mitoses; and frequent admixed melanophages. Criteria for epithelioid blue nevus were: primarily intradermal location; medium-sized epithelioid melanocytes with vesicular chromatin and heavily pigmented cytoplasm; larger epithelioid melanocytes with prominent nucleoli and abundant less pigmented cytoplasm; spindled dendritic melanocytes; frequent admixed melanophages; and no or occasional mitoses. Criteria for Spitz nevi and blue nevi were as described. ${ }^{23}$ The study was approved by and performed in accordance with Miraca Life Sciences IRB protocol requirements.

A board certified dermapathologist (JR) reviewed an H\&E slide of each tumor sample and determined the percentage of tumor nuclei within the area to be macrodissected, with a minimum of $70 \%$ tumor nuclei present. Each specimen was macrodissected and DNA was extracted using the Qiagen FFPE Tissue DNA Kit (Qiagen). DNA was then amplified with primers flanking exons 4 and 5 of GNAQ and GNA11 and exons 2 and 3 of HRAS.

The M13-linked primer sequences used are as follows (M13 linkers written in lower case): exon 4 GNAQ forward 3 '-tgtaaaacgacggccagtcTGGTGTGATGGTGTCACTGACAT-5'; exon 4 GNAQ reverse $3^{\prime}$-aacagctatgaccatgAAGGCATAAAAGCTGGGAAAT -5'; exon 5 GNAQ forward 3'-tgtaaaacgacggccagtcCC CACACCCTACTTTCTATCATTTAC-5'; exon 5 GN $A Q$ reverse $3^{\prime}$-aacagctatgaccatgTTTTCCCTAAGTT TGTAAGTAGTGC-5'; exon 4 GNA11 forward 3'-tgtaaaacgacggccagtcGTGCTGTGTCCCTGTCCTG-5'; exon 4 GNA11 reverse $3^{\prime}$-aacagctatgaccatg GGCAAATGAGCCTCTCAGTG-5'; exon 5 GNA11 forward 3 '-tgtaaaacgacggccagtcgtcCTGGGATTGCAG ATTG-5'; exon 5 GNA11 reverse $3^{\prime}$-aacagctatgac catgCCACCAGGACTTGGTCGTAT-5' ${ }^{\prime}$; exon 2 HRAS forward $3^{\prime}$-tgtaaaacgacggccagtcAGGAGACCCTGTAG GAGGA-5'; exon 2 HRAS reverse 3 '-aacagctatgacc atgCCTATCCTGGCTGTGTCCTG-5'; exon 3 HRAS forward 3 '-tgtaaaacgacggccagtcAGAGGCTGGCTGT GTGAACT-5'; and exon 3 HRAS reverse $3^{\prime}$-aacagc tatgaccatgTCACGGGGTTCACCTGTACT-5'.

PCR amplification was performed with $0.4 \mu \mathrm{M}$ forward and reverse primer, high fidelity Taq polymerase (Invitrogen), and buffer supplied by the manufacturer in $25 \mu$ l reactions.

Touchdown PCR thermocycle conditions are as follows: $94{ }^{\circ} \mathrm{C}$ for $2 \mathrm{~min}$ followed by three cycles of $94{ }^{\circ} \mathrm{C}$ for $30 \mathrm{~s}, 60{ }^{\circ} \mathrm{C}$ for $30 \mathrm{~s}, 68^{\circ} \mathrm{C}$ for $1 \mathrm{~min}$ followed by three cycles of $94{ }^{\circ} \mathrm{C}$ for $30 \mathrm{~s}, 59^{\circ} \mathrm{C}$ for $30 \mathrm{~s}, 68^{\circ} \mathrm{C}$ for $1 \mathrm{~min}$ followed by three cycles of $94^{\circ} \mathrm{C}$ for $30 \mathrm{~s}$, $58{ }^{\circ} \mathrm{C}$ for $30 \mathrm{~s}, 68^{\circ} \mathrm{C}$ for 1 min followed by 30 cycles of $94{ }^{\circ} \mathrm{C}$ for $30 \mathrm{~s}, 57^{\circ} \mathrm{C}$ for $30 \mathrm{~s}, 68^{\circ} \mathrm{C}$ for $1 \mathrm{~min}$. Amplicons of interest were purified using AMPure (Agencourt). Purified amplicons were cycle sequenced using M13 primers and BigDye Terminator 1.1 (Applied Biosystems) per the manufacturer's instructions and purified with Agencourt CleanSeq kit (Beckman Coulter). M13 primer sequences used are as follows: M13 forward 3'-TGTAAAACGACGGCCAGTC-5' ${ }^{\prime}$; and M13 reverse $3^{\prime}$-AACAGCTATGACCATG- $5^{\prime}$. Cycle sequencing products were resolved using an ABI 3730 instrument (Applied Biosystems) and data were analyzed by a board certified molecular geneticist using Mutation Surveyor (Soft Genetics).

\section{Results}

Patient demographics and genotyping are summarized in Table 2. 
Table 2 Patient demongraphics and mutational analysis

\begin{tabular}{|c|c|c|c|c|c|c|c|c|c|c|}
\hline \multirow{2}{*}{$\begin{array}{l}\text { Case } \\
\text { number }\end{array}$} & \multirow[b]{2}{*}{ Age } & \multirow[b]{2}{*}{ Sex } & \multirow[b]{2}{*}{ Site } & \multirow[b]{2}{*}{ Type } & \multicolumn{2}{|r|}{$G N A Q$} & \multicolumn{2}{|r|}{ GNA11 } & & $A S$ \\
\hline & & & & & $e \times 4$ & ex 5 & $e x 4$ & ex 5 & $e \times 2$ & ex 3 \\
\hline 1 & 40 & $\mathrm{~F}$ & $\mathrm{R}$ upper arm & $\mathrm{CBN}$ & WT & WT & WT & WT & WT & WT \\
\hline 2 & 14 & $\mathrm{M}$ & L dorsal foot & $\mathrm{CBN}$ & WT & Q209L 626A > T & WT & WT & WT & WT \\
\hline 3 & 6 & $\mathrm{~F}$ & Buttocks & $\mathrm{CBN}$ & WT & $\widehat{Q} 209 \mathrm{~L} 626 \mathrm{~A}>\mathrm{T}$ & WT & WT & WT & WT \\
\hline 4 & 51 & $\mathrm{~F}$ & Scalp & $\mathrm{CBN}$ & WT & $\widehat{\mathrm{WT}}$ & WT & WT & WT & WT \\
\hline 5 & 43 & $\mathrm{M}$ & Scalp & $\mathrm{CBN}$ & WT & $\mathrm{Q} 209 \mathrm{~L} 626 \mathrm{~A}>\mathrm{T}$ & WT & WT & WT & WT \\
\hline 6 & 53 & $\mathrm{M}$ & Intergluteal fold & $\mathrm{CBN}$ & WT & Q209L 626A > T & WT & WT & WT & WT \\
\hline 7 & 18 & $\mathrm{~F}$ & Buttocks & $\mathrm{CBN}$ & WT & $\mathrm{Q} 209 \mathrm{~L} 626 \mathrm{~A}>\mathrm{T}$ & WT & WT & WT & WT \\
\hline 8 & 13 & $\mathrm{~F}$ & Buttocks & $\mathrm{CBN}$ & WT & $\widehat{\mathrm{WT}}$ & WT & Q209L 626A > T & WT & WT \\
\hline 9 & 51 & $\mathrm{~F}$ & Buttocks & $\mathrm{CBN}$ & WT & Q209L 626A > T & WT & WT & WT & WT \\
\hline 10 & 7 & $\mathrm{~F}$ & Buttocks & CBN & WT & $\mathrm{Q} 209 \mathrm{~L} 626 \mathrm{~A}>\mathrm{T}$ & WT & WT & WT & WT \\
\hline 11 & 14 & $\mathrm{M}$ & $\mathrm{R}$ upper thigh & $\mathrm{CBN}$ & WT & $\widehat{\mathrm{Q}} 209 \mathrm{~L} 626 \mathrm{~A}>\mathrm{T}$ & WT & WT & WT & WT \\
\hline 12 & 36 & $\mathrm{~F}$ & Buttocks & $\mathrm{CBN}$ & WT & Q209L 626A > T & WT & WT & WT & WT \\
\hline 13 & 11 & $\mathrm{~F}$ & Back & $\mathrm{CBN}$ & WT & $\mathrm{Q} 209 \mathrm{~L} 626 \mathrm{~A}>\mathrm{T}$ & WT & WT & WT & WT \\
\hline 14 & 63 & $\mathrm{~F}$ & Scalp & $\mathrm{CBN}$ & WT & $\mathrm{Q} 209 \mathrm{~L} 626 \mathrm{~A}>\mathrm{T}$ & WT & WT & WT & WT \\
\hline 15 & 15 & $\mathrm{~F}$ & $\mathrm{R}$ dorsal hand & $\mathrm{CBN}$ & WT & $\mathrm{Q} 209 \mathrm{~L} 626 \mathrm{~A}>\mathrm{T}$ & WT & WT & WT & WT \\
\hline 16 & 18 & $\mathrm{M}$ & $\mathrm{R}$ upper arm & $\mathrm{CBN}$ & WT & Q209L $626 \mathrm{~A}>\mathrm{T}$ & WT & WT & WT & WT \\
\hline 17 & 30 & $\mathrm{~F}$ & Buttocks & $\mathrm{CBN}$ & WT & $\mathrm{Q} 209 \mathrm{~L} 626 \mathrm{~A}>\mathrm{T}$ & WT & WT & WT & WT \\
\hline 18 & 16 & $\mathrm{~F}$ & Buttocks & $\mathrm{CBN}$ & WT & $\mathrm{Q} 209 \mathrm{~L} 626 \mathrm{~A}>\mathrm{T}$ & WT & WT & WT & WT \\
\hline 19 & 78 & $\mathrm{~F}$ & Scalp & $\mathrm{BN}$ & WT & $\widehat{Q} 209 \mathrm{~L} 626 \mathrm{~A}>\mathrm{T}$ & WT & WT & WT & WT \\
\hline 20 & 26 & $\mathrm{~F}$ & Scalp & $\mathrm{BN}$ & WT & $\mathrm{Q} 209 \mathrm{~L} 626 \mathrm{~A}>\mathrm{T}$ & WT & WT & WT & WT \\
\hline 21 & 35 & $\mathrm{~F}$ & $\mathrm{R}$ hand & $\mathrm{BN}$ & WT & Q209L 625_626CA > TT & ND & ND & ND & ND \\
\hline 22 & 75 & $\mathrm{M}$ & Scalp & $\mathrm{BN}$ & WT & $\widehat{\mathrm{Q}} 209 \mathrm{~L} 626 \overline{\mathrm{A}}>\mathrm{T}$ & WT & WT & WT & WT \\
\hline 23 & 15 & $\mathrm{M}$ & $\mathrm{R}$ upper arm & $\mathrm{BN}$ & WT & $\mathrm{Q} 209 \mathrm{~L} 626 \mathrm{~A}>\mathrm{T}$ & WT & WT & WT & WT \\
\hline 24 & 13 & $\mathrm{M}$ & $\mathrm{R}$ dorsal hand & $\mathrm{BN}$ & WT & $\mathrm{Q} 209 \mathrm{~L} 626 \mathrm{~A}>\mathrm{T}$ & ND & ND & ND & ND \\
\hline 25 & 17 & $\mathrm{M}$ & $\mathrm{R}$ forearm & $\mathrm{BN}$ & WT & Q209L 625_626CA > TT & WT & WT & WT & WT \\
\hline 26 & 46 & $\mathrm{M}$ & $\mathrm{L}$ wrist & $\mathrm{BN}$ & WT & WT & WT & WT & WT & WT \\
\hline 27 & 40 & $\mathrm{~F}$ & $\mathrm{R}$ hand & $\mathrm{BN}$ & WT & $\mathrm{Q} 209 \mathrm{~L} 626 \mathrm{~A}>\mathrm{T}$ & WT & WT & WT & WT \\
\hline 28 & 72 & $\mathrm{~F}$ & Scalp & $\mathrm{BN}$ & WT & Q209L 626A > T & WT & WT & WT & WT \\
\hline 29 & 37 & $\mathrm{M}$ & Scalp & $\mathrm{BN}$ & WT & $\mathrm{Q} 209 \mathrm{~L} 626 \mathrm{~A}>\mathrm{T}$ & WT & WT & WT & WT \\
\hline 30 & 47 & $\mathrm{~F}$ & $\mathrm{R}$ dorsal forearm & $\mathrm{BN}$ & WT & $\mathrm{Q} 209 \mathrm{~L} 626 \mathrm{~A}>\mathrm{T}$ & WT & WT & WT & WT \\
\hline 31 & 12 & $\mathrm{~F}$ & L ankle & $\mathrm{DPN}$ & WT & WT & WT & WT & ND & WT \\
\hline 32 & 21 & $\mathrm{M}$ & $\mathrm{R}$ upper arm & $\mathrm{DPN}$ & WT & WT & ND & ND & ND & ND \\
\hline 33 & 23 & $\mathrm{M}$ & Chest & $\mathrm{DPN}$ & WT & WT & WT & WT & WT & WT \\
\hline 34 & 30 & $\mathrm{M}$ & Chest & $\mathrm{DPN}$ & WT & WT & WT & WT & WT & Q61L 182A $>$ T \\
\hline 35 & 26 & $\mathrm{~F}$ & R scapula & $\mathrm{DPN}$ & WT & WT & WT & WT & G13R 37G $>C$ & WT \\
\hline 36 & 27 & $\mathrm{~F}$ & $\mathrm{R}$ calf & $\mathrm{DPN}$ & WT & WT & WT & WT & ND & WT \\
\hline 37 & 25 & $\mathrm{~F}$ & Back & DPN & WT & WT & WT & WT & WT & WT \\
\hline 38 & 72 & $\mathrm{M}$ & $\mathrm{R}$ upper arm & $\mathrm{DPN}$ & WT & WT & WT & WT & WT & WT \\
\hline 39 & 39 & $\mathrm{~F}$ & L shin & $\mathrm{DPN}$ & WT & WT & WT & WT & WT & WT \\
\hline 40 & 36 & $\mathrm{M}$ & $\mathrm{L}$ forearm & DPN & WT & WT & WT & WT & ND & WT \\
\hline 41 & 23 & $\mathrm{~F}$ & $\mathrm{R}$ upper arm & $\mathrm{DPN}$ & WT & WT & WT & WT & WT & ND \\
\hline 42 & 48 & $\mathrm{M}$ & $\mathrm{R}$ cheek & $\mathrm{DPN}$ & WT & WT & WT & WT & WT & WT \\
\hline 43 & 17 & $\mathrm{~F}$ & $\mathrm{~L}$ forearm & $\mathrm{DPN}$ & WT & WT & WT & WT & WT & WT \\
\hline 44 & 16 & $\mathrm{~F}$ & Scalp & $\mathrm{DPN}$ & WT & WT & WT & WT & ND & ND \\
\hline 45 & 77 & $\mathrm{~F}$ & L thigh & $\mathrm{DPN}$ & WT & WT & ND & ND & ND & ND \\
\hline 46 & 32 & $\mathrm{~F}$ & L lower leg & $\mathrm{DPN}$ & WT & WT & ND & ND & ND & ND \\
\hline 47 & 32 & $\mathrm{~F}$ & R temple & $\mathrm{DPN}$ & WT & WT & WT & WT & WT & WT \\
\hline 48 & 27 & $\mathrm{~F}$ & Groin & $\mathrm{DPN}$ & WT & WT & WT & WT & WT & WT \\
\hline 49 & 59 & $\mathrm{M}$ & Lower leg & $\mathrm{DPN}$ & WT & WT & WT & WT & WT & WT \\
\hline 50 & 24 & $\mathrm{~F}$ & $\mathrm{R}$ nasal sidewall & $\mathrm{DPN}$ & WT & WT & WT & WT & WT & WT \\
\hline 51 & 35 & $\mathrm{~F}$ & Upper forehead & $\mathrm{DPN}$ & WT & WT & WT & WT & WT & WT \\
\hline 52 & 30 & $\mathrm{~F}$ & $\mathrm{R}$ lower arm & $\mathrm{DPN}$ & WT & WT & WT & WT & WT & WT \\
\hline 53 & 48 & $\mathrm{M}$ & R scapula & $\mathrm{DPN}$ & WT & WT & WT & WT & WT & WT \\
\hline 54 & 28 & $\mathrm{~F}$ & Back & $\mathrm{DPN}$ & WT & WT & ND & ND & ND & WT \\
\hline 55 & 14 & $\mathrm{M}$ & Back & $\mathrm{DPN}$ & WT & WT & ND & ND & ND & WT \\
\hline 56 & 47 & $\mathrm{~F}$ & $\mathrm{~L}$ ankle & $\mathrm{DPN}$ & WT & WT & ND & WT & ND & ND \\
\hline 57 & NA & NA & NA & $\mathrm{DPN}$ & WT & WT & WT & WT & WT & WT \\
\hline 58 & 34 & $\mathrm{M}$ & L cheek & $\mathrm{DPN}$ & WT & WT & ND & WT & ND & ND \\
\hline 59 & 19 & $\mathrm{M}$ & Back & $\mathrm{DPN}$ & WT & WT & WT & WT & WT & WT \\
\hline 60 & 50 & $\mathrm{M}$ & R thigh & $\mathrm{DPN}$ & WT & WT & WT & WT & WT & ND \\
\hline 61 & 20 & $\mathrm{~F}$ & R cheek & $\mathrm{DPN}$ & WT & WT & WT & WT & WT & WT \\
\hline 62 & 35 & $\mathrm{~F}$ & L flank & $\mathrm{DPN}$ & WT & WT & WT & WT & WT & ND \\
\hline 63 & 24 & $\mathrm{~F}$ & Back & $\mathrm{DPN}$ & WT & WT & WT & WT & WT & WT \\
\hline 64 & 42 & $\mathrm{M}$ & $\mathrm{R}$ arm & DPN & WT & WT & WT & WT & WT & WT \\
\hline 65 & 18 & $\mathrm{~F}$ & $\mathrm{R}$ thigh & $\mathrm{DPN}$ & WT & WT & WT & WT & ND & ND \\
\hline 66 & 18 & $\mathrm{~F}$ & Back & $\mathrm{DPN}$ & WT & WT & WT & WT & WT & WT \\
\hline 67 & 46 & $\mathrm{~F}$ & $\mathrm{R}$ axillary line & $\mathrm{DPN}$ & WT & WT & WT & WT & WT & WT \\
\hline 68 & 34 & $\mathrm{~F}$ & L lower leg & $\mathrm{DPN}$ & WT & WT & WT & WT & ND & ND \\
\hline 69 & 37 & $\mathrm{~F}$ & L upper arm & $\mathrm{EBN}$ & WT & WT & WT & WT & ND & ND \\
\hline 70 & 17 & $\mathrm{M}$ & L leg & $\mathrm{EBN}$ & WT & WT & WT & WT & WT & WT \\
\hline 71 & 67 & $\mathrm{~F}$ & $\mathrm{R}$ dorsal hand & $\mathrm{EBN}$ & WT & Q209L 626A >T & WT & WT & WT & WT \\
\hline
\end{tabular}


Table 2 (Continued)

\begin{tabular}{|c|c|c|c|c|c|c|c|c|c|c|}
\hline \multirow[b]{2}{*}{$\begin{array}{l}\text { Case } \\
\text { number }\end{array}$} & \multirow[b]{2}{*}{ Age } & \multirow[b]{2}{*}{ Sex } & \multirow[b]{2}{*}{ Site } & \multirow[b]{2}{*}{ Type } & \multicolumn{2}{|r|}{$G N A Q$} & \multicolumn{2}{|r|}{ GNA11 } & \multicolumn{2}{|c|}{ HRAS } \\
\hline & & & & & $e x 4$ & ex 5 & ex 4 & ex 5 & ex 2 & $e \times 3$ \\
\hline 72 & 46 & M & Back & EBN & WT & WT & WT & WT & WT & WT \\
\hline 73 & 66 & M & $\mathrm{R}$ shoulder & EBN & WT & WT & ND & ND & ND & ND \\
\hline 74 & 32 & NA & $\mathrm{R}$ forearm & EBN & WT & WT & ND & WT & ND & WT \\
\hline 75 & 62 & $\mathrm{M}$ & Abdomen & EBN & WT & WT & WT & WT & WT & WT \\
\hline 76 & 64 & $\mathrm{M}$ & L shoulder & EBN & WT & WT & WT & WT & WT & WT \\
\hline 77 & 16 & $\mathrm{M}$ & R prearicular & EBN & WT & ND & WT & WT & ND & ND \\
\hline 78 & 41 & $\mathrm{~F}$ & $\mathrm{~L}$ forearm & EBN & WT & Q209L 626A $>$ T & ND & WT & ND & ND \\
\hline 79 & 88 & $\mathrm{~F}$ & L upper arm & EBN & WT & WT & WT & WT & WT & ND \\
\hline 80 & 21 & $\mathrm{~F}$ & $\mathrm{R}$ upper arm & $\mathrm{SN}$ & WT & WT & WT & WT & WT & ND \\
\hline 81 & 19 & $\mathrm{~F}$ & Buttocks & SN & WT & WT & WT & WT & WT & WT \\
\hline 82 & 36 & $\mathrm{M}$ & Chest & $\mathrm{SN}$ & ND & WT & ND & ND & WT & WT \\
\hline 83 & 34 & $\mathrm{~F}$ & Back & $\mathrm{SN}$ & WT & WT & WT & WT & WT & Q61L 182A > T \\
\hline 84 & 17 & $\mathrm{~F}$ & L lower leg & $\mathrm{SN}$ & WT & WT & WT & WT & WT & WT \\
\hline 85 & 28 & $\mathrm{~F}$ & L thigh & $\mathrm{SN}$ & WT & WT & WT & WT & WT & WT \\
\hline 86 & 18 & $\mathrm{~F}$ & $\mathrm{~L}$ arm & $\mathrm{SN}$ & WT & WT & WT & WT & WT & WT \\
\hline 87 & 37 & $\mathrm{~F}$ & Back & $\mathrm{SN}$ & WT & WT & WT & WT & WT & WT \\
\hline 88 & 33 & $\mathrm{~F}$ & Back & $\mathrm{SN}$ & WT & WT & WT & WT & G13R $37 \mathrm{G}>\mathrm{C}$ & WT \\
\hline 89 & 55 & $\mathrm{M}$ & $\mathrm{R}$ arm & SN & WT & WT & WT & WT & WT & Q61L 182A > T \\
\hline 90 & 25 & $\mathrm{~F}$ & Back & $\mathrm{SN}$ & WT & WT & WT & WT & WT & WT \\
\hline 91 & 28 & $\mathrm{~F}$ & Chest & $\mathrm{SN}$ & WT & WT & WT & WT & WT & WT \\
\hline 92 & 29 & $\mathrm{~F}$ & Buttocks & SN & WT & WT & WT & WT & WT & WT \\
\hline 93 & 55 & $\mathrm{~F}$ & L thigh & SN & WT & WT & WT & WT & WT & WT \\
\hline 94 & 49 & $\mathrm{M}$ & $\mathrm{L}$ forearm & SN & WT & WT & WT & WT & WT & WT \\
\hline 95 & 5 & $\mathrm{~F}$ & R ankle & SN & WT & WT & WT & WT & WT & WT \\
\hline 96 & 5 & $\mathrm{~F}$ & R elbow & SN & WT & WT & WT & WT & WT & WT \\
\hline 97 & 24 & $\mathrm{M}$ & L shoulder & $\mathrm{SN}$ & WT & WT & WT & WT & WT & WT \\
\hline 98 & 5 & $\mathrm{~F}$ & R knee & SN & WT & WT & WT & WT & WT & WT \\
\hline 99 & 12 & $\mathrm{~F}$ & L shoulder & $\mathrm{SN}$ & WT & WT & WT & WT & WT & WT \\
\hline 100 & 6 & $\mathrm{M}$ & L thigh & $\mathrm{SN}$ & WT & WT & WT & WT & WT & ND \\
\hline 101 & 14 & $\mathrm{M}$ & Back & $\mathrm{SN}$ & WT & WT & WT & WT & G13R 37G >C & WT \\
\hline 102 & 27 & $\mathrm{~F}$ & Lower leg & $\mathrm{SN}$ & WT & WT & WT & WT & WT & WT \\
\hline 103 & 53 & $\mathrm{~F}$ & $\mathrm{R}$ lower leg & $\mathrm{SN}$ & WT & WT & WT & WT & WT & WT \\
\hline 104 & 49 & $\mathrm{~F}$ & R knee & $\mathrm{SN}$ & WT & ND & ND & WT & G12S $34 \mathrm{G}>\mathrm{A}$ & WT \\
\hline 105 & 5 & $\mathrm{~F}$ & Scalp & $\mathrm{SN}$ & WT & WT & WT & WT & WT & WT \\
\hline 106 & 21 & $\mathrm{~F}$ & L antecubital fossa & SN & WT & WT & WT & WT & WT & WT \\
\hline 107 & 55 & $\mathrm{~F}$ & R medial knee & $\mathrm{SN}$ & WT & WT & ND & ND & WT & WT \\
\hline 108 & 50 & $\mathrm{~F}$ & $\mathrm{R}$ temple & $\mathrm{SN}$ & WT & WT & WT & WT & WT & WT \\
\hline 109 & 16 & $\mathrm{~F}$ & $\mathrm{R}$ thigh & $\mathrm{SN}$ & WT & WT & WT & WT & WT & WT \\
\hline
\end{tabular}

Abbreviations: CBN, cellular blue nevus; BN, common blue nevus; DPN, deep penetrating nevus; EBN, epithelioid blue nevus; SN, Spitz nevus; WT, wild type; NA, not available; ND, not done.

\section{Deep Penetrating Nevi}

A total of 38 deep penetrating nevi were retrieved from our files. Of these patients, 24 were female, 13 were male, (gender was not available for 1 case), for a $\mathrm{M}: \mathrm{F}$ ratio of approximately $1: 2$. As in the literature, the most common site was the extremities (11 on the legs, 7 on the arms), followed by the trunk (12) and head and neck (7). We could not retrieve the site data for one patient. Ages ranged from 12 to 77 years (mean age: 33 years, median age: 30 years).

Six percent of deep penetrating nevi $(2 / 32)$ contained HRAS mutations (cases 34 and 35), 1 in exon 2 (p.G13R, c.37G $>$ C point mutation) and 1 in exon 3 (p.Q61L, c.182A $>\mathrm{T}$ point mutation). Both cases displayed the typical features of a deep penetrating nevus, with primarily intradermal location, plexiform growth in the dermis, epithelioid melanocytes with vesicular chromatin and abundant dusky cytoplasm, extension down neurovascular bundles, and admixed melanophages (Figure 1). Intranuclear pseudo-inclusions (frequently seen in deep penetrating nevi) were also present (Figures 1c and f). No significant pleomorphism was identified in either case; however, case 34 contained a mitosis (Figure 1f). In comparison to all the other deep penetrating nevi tested, no distinctive histological features distinguished these two cases from the others in this study nor from the diagnostic criteria delineated in the literature for deep penetrating nevi. None of the deep penetrating nevi analyzed contained GNAQ or GNA11 mutations (0/38 and $0 / 33$, respectively).

\section{Epithelioid Blue Nevi}

Epithelioid blue nevi were the least common of the nevic subtypes in our study, with just 11 confirmed cases in our files within the 6 years of retrieved data. 

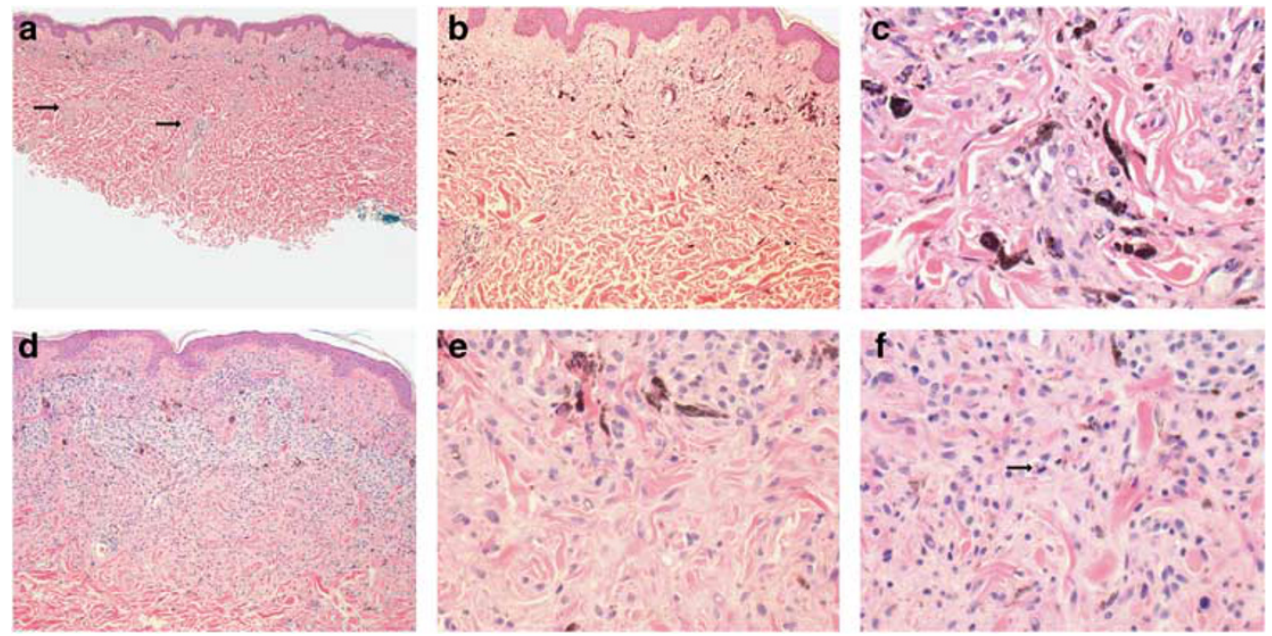

Figure 1 Two HRAS-mutated deep penetrating nevi. (a-c): case 35, (d-f): case 34. (a) Wedge shape, extension along neurovascular bundles (arrows). (b) Abundant admixed melanophages, plexiform growth, neurovascular extension. (c) Melanocytes with abundant finely pigmented cytoplasm. (d) Prominent plexiform growth, admixed melanophages. (e) Melanocytes with indistinct nucleoli, abundant finely pigmented cytoplasm. (f) Atypical mitosis (arrow).
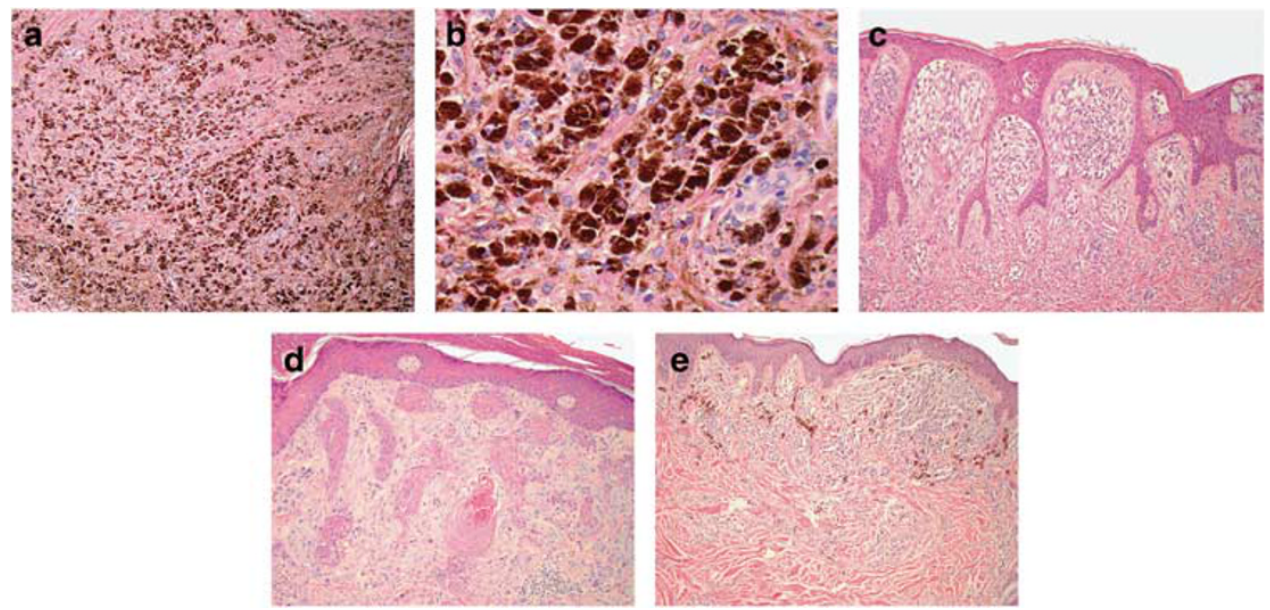

Figure 2 GNAQ-mutated epithelioid blue nevi, and HRAS-mutated Spitz nevi. (a, b): case 78 (epithelioid blue nevus). (a) Heavily pigmented melanocytic proliferation, with pigmented round epithelioid melanocytes, pigmented dendritic melanocytes, and numerous melanophages. (b) Scattered larger epithelioid cells with abundant less pigmented cytoplasm, admixed with round epithelioid melanocytes with prominent nucleoli and heavily pigmented cytoplasm, pigmented dendritic melanocytes, and melanophages. (c): case 101 (Spitz nevus); (d): case 104 (Spitz nevus); and (e): case 83 (Spitz nevus). (c) Compound cellular Spitz nevus. (d) Compound cellular Spitz nevus with atypia. (e) Heavily pigmented Spitz nevus.

The M:F ratio was 1.5:1 (4 female, 6 male; gender was not available for 1 case). The age ranged from 16 to 88 years (mean age: 49 years, median age: 46 years). The most common anatomical location was the extremities (4 on the arms, 1 on the leg), followed by the trunk (4).

Twenty percent of epithelioid blue nevi $(2 / 10)$ demonstrated a GNAQ mutation, whereas none (0/10) displayed a GNA11 mutation. Both GNAQ mutations were exon 5 p.Q209L, c.626A $>$ T point mutations. None of the epithelioid blue nevi (0/6) contained a HRAS mutation. No distinctive histological features separated the epithelioid blue nevi with GNAQ mutations from those without a mutation (Figures 2a and b).

\section{Spitz Nevi}

Thirty Spitz nevi were retrieved from our files. Six patients were male, and 24 were female, for a $\mathrm{M}: \mathrm{F}$ ratio of 1:4. The age ranged from 5 to 55 years (mean age: 28 years, median age: 27 years). The most common site was the extremities (6 on the arms, 11 on the legs), followed by the trunk (11), and then the head and neck (2).

HRAS mutation was found in $17 \%(5 / 30)$ of Spitz nevi. Three were in exon 2 (p.G13R c.37G $>$ C point mutations), and two were in exon 3 (p.Q61L c.182A $>$ T point mutations). None demonstrated GNAQ or GNA11 mutations (0/30). Histological evaluation of the Spitz nevi with HRAS mutations 
revealed some findings similar to the features previously characterized in Spitz nevi with HRAS mutations; ${ }^{15,16}$ however, distinct differences were also noted. As mentioned previously, the HRASmutated Spitz nevi in our study also showed delicate infiltration of the dermal collagen by melanocytes at the base of the lesion. This finding was not specific to the HRAS-mutated Spitz nevi in our study, as 8 of the 25 non-mutated Spitz nevi also displayed this pattern of growth at the base. Unlike in the previous studies, which highlighted primarily intradermal location as a distinctive feature, the majority of the HRAS-mutated Spitz nevi in our study were compound (3), Figures 2c and d, rather than purely intradermal (1), and only 2 showed stromal desmoplasia with single cells between collagen bundles. Of note, 4 of the 25 non-mutated Spitz nevi were hypocellular and 7 of them showed desmoplasia. In addition, the majority of the five HRAS-mutated Spitz nevi were cellular (Figures 2c and d), while just two displayed the hypocellular appearance described as a distinctive feature by van Engen-van Grunsven et al. ${ }^{16}$ One of the HRASmutated Spitz nevi was also heavily pigmented (Figure 2e), unlike the hypopigmentation described in the previous study. One of the HRAS-mutated Spitz nevi displayed atypical features, including focal pleomorphism and occasional mitoses (case 104, Figure 2d).

\section{Blue Nevi}

Thirty blue nevi were analyzed. Of these, 19 were female and 11 were male, for a M:F ratio of 1:1.7. The age range was from 6 to 78 years (mean age: 33 years, median age: 32.5 years). The most common site was the buttocks (9), followed by the scalp (8), extremities ( 6 on the arm, 1 on the leg), and acral (4 on the hands, 1 on the foot).

The majority of blue nevi $(87 \% ; 26 / 30)$ displayed GNAQ mutations, all in exon 5. Most of the mutations were p.Q209L, c.626A $>$ T point mutations, with the exception of two cases that contained novel p.Q209L, c.625_626CA > TT double mutations (cases 23 and 27). A GNA11 mutation was found in one blue nevus $(1 / 28 ; 4 \%)$, which was an exon 5 p.G209L, c.626A $>$ T point mutation. None of the blue nevi samples tested contained a HRAS mutation $(0 / 28)$.

\section{Discussion}

We have analyzed deep penetrating nevi and epithelioid blue nevi for GNAQ, GNA11, and HRAS mutations, to further characterize and compare these unusual melanocytic neoplasms to other epithelioid and primarily intradermal melanocytic nevi, namely Spitz nevi and blue nevi. Approximately one-third of Spitz nevi show copy number gains in chromosome $11 \mathrm{p}$, with $15 \%$ harboring an activating HRAS mutation. Previous to this study, no other nevic variants other than Spitz nevi have demonstrated HRAS mutations. Although HRAS mutations have been reported in melanoma, it appears to be rare. The presence of a GNAQ or GNA11 mutation characterizes the majority of blue nevi ( $>85 \%$ ). The distinct histological features of deep penetrating nevi have been detailed previously in the literature; however, molecular analysis of these lesions has been limited. Due to their primarily intradermal location and pigmentation, classification as a variant of a blue nevus, particularly an epithelioid blue nevus, has been proposed, and consideration has also been given to classification of deep penetrating nevi within the Spitz nevus category. This study is the first to analyze deep penetrating nevi for HRAS mutations, the largest series to assess deep penetrating nevi for GNAQ and GNA11 mutations, the first to test blue nevi for HRAS mutations, and the first to analyze epithelioid blue nevi for both HRAS and GNAQ and GNA11 mutations. The presence of GNAQ mutations and the absence of HRAS mutations in the epithelioid blue nevi in our study provides molecular support for the classification of these tumors as true variants of blue nevi, while the presence of HRAS mutations and the absence of GNAQ or GNA11 mutations in deep penetrating nevi suggest similarity to Spitz nevi rather than blue nevi. As both deep penetrating nevi and epithelioid blue nevi can pose a diagnostic challenge due to overlapping clinical as well as histological findings, and both have shown the propensity for locoregionally aggressive behavior on rare occasion, the identification of mutations in HRAS and GNAQ could be used as valuable diagnostic tools to help differentiate these nevi from each other as well as from melanoma.

HRAS belongs to the RAS gene family, which also includes NRAS and KRAS. Although NRAS is mutated in a significant subset of melanomas (approximately 20\%), of those analyzed in the literature, HRAS mutation in melanoma appears to be rare. ${ }^{15,17-19,24-27}$ In addition, all published cases of HRAS-mutated Spitz tumors with clinical followup have behaved in a benign fashion, suggesting that the presence of a HRAS mutation in a lesion with Spitzoid morphology is a good prognostic indicator. ${ }^{15-17}$ The presence of an activating HRAS mutation in $6 \%$ of deep penetrating nevi in our study sheds new light on the molecular pathogenesis of these tumors. As in both benign Spitz nevi with HRAS mutations, and BRAF mutations in a majority of benign melanocytic nevi, a RAS mutation is not sufficient to promote malignant change within a nevus. It is interesting to note that one of the two HRAS-mutated deep penetrating nevi in our study had atypical features, namely the presence of an atypical deep mitosis. As in Spitz nevi with atypical features, the presence of a HRAS mutation may support a benign prognosis, a hypothesis which will need to be tested further. 
Interestingly, our study suggests a broader array of histological findings in HRAS-mutated Spitz nevi (and consequent difficulty in predicting the presence of a HRAS mutation based on morphology) than in previous studies. Of the features listed previously in the literature as distinct to Spitz nevi with HRAS mutations (single cells between collagen bundles, infiltrative base, desmoplasia, predominantly intradermal location, and relatively low cellularity (the latter seen in $\sim 42 \%$ of cases), ${ }^{15,16}$ the only feature we found to overlap with the HRASmutated Spitz nevi in our study was an infiltrative base with dispersion of melanocytes around reticular collagen bundles. This finding, however, was not specific to HRAS-mutated Spitz nevi in our study, as a significant subset of non-HRAS-mutated Spitz nevi also displayed this growth pattern (8 of $25)$. In addition, while 2 of the 5 HRAS-mutated Spitz nevi in our study demonstrated desmoplasia with single cells between collagen bundles, 7 (28\%) of non-HRAS-mutated Spitz nevi in our study also showed this growth pattern. Although it is possible that these previously mentioned Spitz nevi had HRAS mutations below the level of assay detection (the lower limit of sensitivity for sanger sequencing is approximately $20 \%$ ), given the relatively high number (32 and $28 \%$ ), it is somewhat unlikely that this is true for all of these cases, as the percentage of tumor to non-tumor present in the area of DNA extraction was high. The majority of the HRAS-mutated Spitz nevi were cellular without desmoplasia, which also argues against the presence of desmoplasia and single-cell dispersion as being features distinctive to HRAS-mutated Spitz nevi.

GNAQ and GNA11 are G-protein alpha subunits that mediate signals between G-coupled protein receptors and their associated downstream pathways. In mice, germline mutation in either of these proteins results in skin hyperpigmentation due to an increase in dermal melanocytes and melanin pigment. ${ }^{22}$ In addition to blue nevi, GNAQ or GNA11 mutations have been found in uveal melanomas as well as malignant blue nevi. ${ }^{10,11}$ Epithelioid blue nevi are associated with Carney Complex and also occur sporadically. In a series of 34 sporadic epithelioid blue nevi with molecular analysis for protein kinase A regulatory subunit $1 \alpha \quad \mathrm{R} 1 \alpha$ (a protein mutated in over half of patients with Carney Complex), none of these lesions harbored R1 $\alpha$ mutations, although $82 \%$ did demonstrate loss of expression by immunohistochemistry. ${ }^{28}$ Other than the previously mentioned study, molecular analysis has been limited on these lesions and is comprised mainly of fluorescence in situ hybridization or BRAF mutation assessment of small case numbers, none of which demonstrated discernible chromosomal aberrations. ${ }^{29,30}$ Our study is the first to analyze epithelioid blue nevi for HRAS, GNAQ, and GNA11 mutations. The presence of GNAQ mutations in a subset of epithelioid blue nevi further confirms categorization of these lesions as true blue nevic variants. Although the percentage of epithelioid blue nevi with GNAQ mutations was less $(20 \%)$ in our study than has been reported in the literature for conventional blue nevi (up to $\sim 80 \%$ ), our sample size for epithelioid blue nevi was small and thus may not be representative of the true incidence of GNAQ and GNA11 mutation in this subcategory of blue nevi. Despite their epithelioid cytomorphology, none demonstrated HRAS mutations. The number of epithelioid blue nevi cases in our series is small, however, and molecular characterization in additional studies of these uncommon nevi would be helpful.

In summary, our data suggest that deep penetrating nevi, in addition to sharing some morphological similarity to Spitz nevi, also share similarities at the molecular level and appear to be unrelated to blue nevi at the molecular level. Although the ability to generalize is hampered by the rarity of epithelioid blue nevi and the consequent small sample size of these nevi in our study, the presence of a detectable GNAQ mutation in this cohort tends to support their classification as blue nevi, despite their unique epithelioid morphological appearance. No distinguishing histopathological features were found to distinguish the HRAS-mutated deep penetrating nevi from the non-HRAS-mutated ones, and in contrast to previous studies, the morphological spectrum of HRAS-mutated Spitz nevi appears to be broader and less distinctive than has been suggested. Mutational as well as cytogenetic analysis of melanocytic tumors with atypical histopathological features (melanocytic tumors of uncertain biological potential) has recently become a frequently utilized tool to aid in the diagnostic and prognostic classification of these tumors, due to the relative ease with which mutational status can be assessed for via targeted sequencing assays. As has been proposed previously for atypical Spitz tumors, mutational analysis for BRAF and HRAS in addition to cytogenetic analysis may also be informative in deep penetrating nevi with atypical features. Future studies will be helpful in assessing the true frequency of HRAS and GNAQ or GNA11 mutations in deep penetrating nevi and epithelioid blue nevi, respectively, as well as studies with long-term clinical follow-up of both mutated and non-mutated forms of these unusual tumors.

\section{Acknowledgements}

We thank Dr Christopher Fletcher (Brigham and Women's Hospital, Harvard Medical School) for critical reading of the paper.

\section{Disclosure/conflict of interest}

The authors declare no conflict of interest. 


\section{References}

1 Seab JA Jr, Graham JH, Helwig EB. Deep penetrating nevus. Am J Surg Pathol 1989;13:39-44.

2 Luzar B, Calonje E. Deep penetrating nevus: a review. Arch Pathol Lab Med 2011;135:321-326.

3 Magro CM, Crowson AN, Mihm MC Jr, et al. The dermal-based borderline melanocytic tumor: a categorical approach. J Am Acad Dermatol 2010;62:469-479.

4 Graham J. Malignant deep penetrating naevus. Poster presentation at the 33rd annual meeting of the American Society of Dermatopathology. J Cutan Pathol 1996;23:76.

5 Abraham RM, Ming ME, Elder DE, et al. An atypical melanocytic lesion without genomic abnormalities shows locoregional metastasis. J Cutan Pathol 2012;39: 21-24.

6 McCalmont TH, Bastian BC. An unconventional deep penetrating melanocytic nevus with microscopic involvement of regional lymph nodes. J Cutan Pathol 2012;39:25-28.

7 Cerroni L, Barnhill R, Elder D, et al. Melanocytic tumors of uncertain malignant potential: results of a tutorial held at the XXIX Symposium of the International Society of Dermatopathology in Graz, October 2008. Am J Surg Pathol 2010;34:314-326.

8 LeBoit P, Burg G, Weedon D, Sarasin A (eds).World Health Organization Classification of Tumours. Pathology and Genetics of Skin Tumours. IARC Press: Lyon, France; 2006, pp 95-99.

9 Maize JC Jr, McCalmont TH, Carlson JA, et al. Genomic analysis of blue nevi and related dermal melanocytic proliferations. Am J Surg Pathol 2005;29: 1214-1220.

10 Van Raamsdonk CD, Bezrookove V, Green G, et al. Frequent somatic mutations of GNAQ in uveal melanoma and blue naevi. Nature 2009;457:599-602.

11 Van Raamsdonk CD, Griewank KG, Crosby MB, et al. Mutations in GNA11 in uveal melanoma. N Engl J Med 2010;363:2191-2199.

12 Carney JA, Stratakis CA. Epithelioid blue nevus and psammomatous melanotic schwannoma: the unusual pigmented skin tumors of the Carney complex. Semin Diagn Pathol 1998;15:216-224.

13 Zembowicz A, Carney JA, Mihm MC. Pigmented epithelioid melanocytoma: a low-grade melanocytic tumor with metastatic potential indistinguishable from animal-type melanoma and epithelioid blue nevus. Am J Surg Pathol 2004;28:31-40.

14 Mandal RV, Murali R, Lundquist KF, et al. Pigmented epithelioid melanocytoma: favorable outcome after 5-year follow-up. Am J Surg Pathol 2009;33:1778-1782.

15 Bastian BC, LeBoit PE, Pinkel D. Mutations and copy number increase of HRAS in Spitz nevi with distinctive histopathological features. Am J Pathol 2000;157: 967-972.

16 van Engen-van Grunsven AC, van Dijk MC, Ruiter DJ, et al. HRAS-mutated Spitz tumors: a subtype of Spitz tumors with distinct features. Am J Surg Pathol 2010;34:1436-1441
17 Da Forno PD, Pringle JH, Fletcher A, et al. BRAF, NRAS and HRAS mutations in spitzoid tumours and their possible pathogenetic significance. $\mathrm{Br}$ J Dermatol 2009;161:364-372.

18 Gill M, Cohen J, Renwick N, et al. Genetic similarities between Spitz nevus and Spitzoid melanoma in children. Cancer 2004;101:2636-2640.

19 van Dijk MC, Bernsen MR, Ruiter DJ. Analysis of mutations in B-RAF, N-RAS, and H-RAS genes in the differential diagnosis of Spitz nevus and spitzoid melanoma. Am J Surg Pathol 2005;29:1145-1151.

20 Takata M, Lin J, Takayanagi S, et al. Genetic and epigenetic alterations in the differential diagnosis of malignant melanoma and spitzoid lesion. Br J Dermatol 2007;156:1287-1294.

21 Blokx WA, van Dijk MC, Ruiter DJ. Molecular cytogenetics of cutaneous melanocytic lesionsdiagnostic, prognostic and therapeutic aspects Histopathology 2010;56:121-132.

22 Van Raamsdonk CD, Fitch KR, Fuchs H, et al. Effects of G-protein mutations on skin color. Nat Genet 2004; 36:961-968.

23 Calonje TBE, Lazar A, McKee PH (eds). McKee’s Pathology of the Skin with Clinical Correlations, 4th edn. Elsevier Saunders: London, UK; 2012, pp 1189-1192.

24 Vidwans SJ, Flaherty KT, Fisher DE, et al. A melanoma molecular disease model. PLoS One 2011;6:e18257.

25 Roychowdhury S, Iyer MK, Robinson DR, et al. Personalized oncology through integrative highthroughput sequencing: a pilot study. Sci Transl Med 2011;3:111ra21.

26 Jiveskog S, Ragnarsson-Olding B, Platz A, et al. N-ras mutations are common in melanomas from sun-exposed skin of humans but rare in mucosal membranes or unexposed skin. J Invest Dermatol 1998;111:757-761.

27 van Elsas A, Zerp SF, van der Flier S, et al. Relevance of ultraviolet-induced N-ras oncogene point mutations in development of primary human cutaneous melanoma. Am J Pathol 1996;149:883-893.

28 Zembowicz A, Knoepp SM, Bei T, et al. Loss of expression of protein kinase a regulatory subunit 1alpha in pigmented epithelioid melanocytoma but not in melanoma or other melanocytic lesions. Am J Surg Pathol 2007;31:1764-1775.

29 Pouryazdanparast $\mathrm{P}$, Newman M, Mafee M, et al. Distinguishing epithelioid blue nevus from blue nevus-like cutaneous melanoma metastasis using fluorescence in situ hybridization. Am J Surg Pathol 2009;33:1396-1400.

30 Battistella M, Prochazkova-Carlotti M, Berrebi D, et al. Two congenital cases of pigmented epithelioid melanocytoma studied by fluorescent in situ hybridization for melanocytic tumors: case reports and review of these recent topics. Dermatology 2010;221:97-106.

31 Lamba S, Felicioni L, Buttitta F, et al. Mutational profile of GNAQQ209 in human tumors. PLoS One 2009;4:e6833.

32 Emley A, Nguyen LP, Yang S, Mahalingam M. Somatic mutations in GNAQ in amelanotic/hypomelanotic blue nevi. Hum Pathol 2011;42:136-140. 